\title{
Guidelines for training in liaison psychiatry
}

\author{
The following guidelines were drawn up by the \\ Executive Committee of the Liaison Psychiatry \\ Group and were approved by the Court of Electors.
}

\section{Introduction}

Liaison psychiatry is particularly concerned with psychiatric disorder as it presents in the medical, surgical and other departments of a general hospital. More specifically it is concerned with the psychiatric accompaniments of physical illness, the somatic presentation of psychiatric illness and the assessment of patients following deliberate self-harm. Experience in these areas is important for psychiatry wherever it is practised.

Liaison psychiatry should be an integral part of all approved training programmes. We do not regard it as a sub-speciality. Valuable experience can, and should, be obtained in child psychiatry and psychogeriatrics as well as in general adult psychiatry. We support the College's statement on General Professional Training which recommends "experience in liaison psychiatry" including "supervised experience in the assessment and management of parasuicide" and the JCHPT recommendation that liaison placements should be available in higher training schemes.

\section{General principles}

1. Liaison psychiatry provides training in the management of clinical problems which are rather different to those seen in mental hospital practice and which require different management skills.

2. Liaison also offers the opportunity for experience of various forms of consultation, which require either the sharing of clinical responsibility with other specialists or acting as a consultant to doctors who retain clinical responsibility.

3. Placements in a general hospital should enable trainees to gain further experience of general medicine and neurology.

\section{Types of experience}

Liaison psychiatry placements should provide trainees with experience and understanding of:

i. symptoms, disabilities and treatment of the common medical illnesses, including the ways in which psychotropic drug regimes need to be modified in physical illness

ii. influence of psychosocial factors on physical health

iii. the range and patterns of psychological response to physical illness, including the distinction between brief affective disturbances and those psychological problems which are persistent and require active intervention

iv. the somatic presentation of psychiatric disorders

v. organic mental disorders: delirium and dementia

vi. alcohol problems in medical patients

vii. psychiatric disorders associated with childbirth.

\section{General skills}

\section{General medical consultations}

The pattern of consultation varies according to the nature of the hospital but wherever possible training should be based on links with a small number of wards and medical teams. Trainees need to learn the general principles, including discussion with the referring clinical team, general practitioners and other informants, recording an opinion in the medical notes and arranging appropriate continuing care. Trainees need to be aware of the particular difficulties of treating patients who do not accept the significance of psychological factors as causes of their complaints.

\section{Deliberate self-harm}

Policies for assessing deliberate self-harm vary from hospital to hospital. The DHSS has recommended that not all patients need be seen by a psychiatrist but psychiatrists have a responsibility to ensure that appropriate psychosocial assessment is made and that those who undertake these routine assessments are able to recognise psychiatric illness and identify those patients who are at high risk of repeating the attempt and killing themselves. The trainee psychiatrist needs to learn:

a. the general principles of organising a service for DSH patients

b. the clinical skills of working closely with medical, nursing and social work colleagues in the assessment of these patients

c. the use of psychological and social interventions. 


\section{Accident and Emergency Department}

Assessing patients in an Accident and Emergency Department provides a valuable opportunity to gain experience of crisis intervention for a wide variety of problems, including acute distress, drug and alcohol problems, the violent patient, and the problems of relatives.

\section{Liaison with specific units}

Close links should be established with certain wards or departments. These enable the psychiatrist to obtain experience of organising regular meetings with the staff to review patients with particular clinical problems, such as those occurring in intensive care, pain clinics, neurology and obstetric and gynaecology units. Staff support groups can also be organised when they are needed.

\section{Timing of liaison experience}

In order to gain adequate experience, we recommend that trainees have a full-time attachment for a minimum period of six months. The attachment should give the trainee the opportunity to develop working relationships with other medical staff and these will be fostered if he attends medical meetings and other non-psychiatric functions.

The stage at which experience in liaison psychiatry is obtained will vary from one training programme to another, according to local practice and service needs. There is much to be said for leaving experience until the end of general professional training when the trainee will have acquired a broad experience of psychiatry. Less experienced trainees require especially close supervision.

It is essential that experience is not confined to the assessment of patients following deliberate self-harm and that trainees are provided with a complete range of experience of psychiatric illness in general hospitals.

Liaison attachments with specialist units require continuity and experience. They are best undertaken by consultants or senior registrars. Trainees will not be able to take on such attachments unless closely supervised by a senior psychiatrist.

\section{Facilities}

Ideally liaison services should be based in a general hospital, with its own accommodation, secretarial services and storage for records. It is an advantage to have beds suitable for the care of patients with both medical and psychiatric disorders. There should be an interview room and a room suitable for seminars and other meetings.

\section{Supervision}

Experience of liaison psychiatry must be adequately supervised. We recommend that one consultant psychiatrist should have administrative responsibility both for the coordination of the liaison service and all training within a rotational scheme. However, it is acknowledged that several consultants may be involved in general hospital consultation liaison and will supervise their own trainees' day-to-day experience. All patients seen by trainees should be regularly discussed, and it is frequently necessary for them also to be seen by a senior psychiatrist. These supervisory sessions will also serve to introduce the trainee to the literature of liaison psychiatry and provide a theoretical basis for clinical practice.

There should be a formal series of lectures or seminars which are available to all registrars in a training scheme. Further teaching experience can be gained if the trainee has the opportunity to present cases to joint meetings or medical grand rounds.

\section{Research}

Research often provides a valuable opportunity to strengthen links which have been made during the course of clinical practice. Collaborative research is to be encouraged and this is more feasible at a senior registrar level. It is essential that any collaborative research programme is well planned in advance, bearing in mind that many psychiatric rating scales have been devised on patients with primary psychiatric illness. If the consultant with supervisory responsibility is not able to undertake supervision himself he should be able to recommend another psychiatrist with the appropriate expertise.

\section{Conclusion}

Liaison psychiatry offers trainees experience of clinical problems and skills which are necessary for psychiatrists wherever they work. Adequate training can only be provided by a well organised service which provides a range of well supervised clinical experience. 